\title{
Predicting the peak growth velocity in the individual child: validation of a new growth model
}

\author{
Iris Busscher • Idsart Kingma $\cdot$ Rob de Bruin • \\ Frits Hein Wapstra · Gijsvertus J. Verkerke • \\ Albert G. Veldhuizen
}

Received: 16 January 2011/Revised: 20 April 2011/ Accepted: 9 May 2011 / Published online: 19 May 2011

(C) The Author(s) 2011. This article is published with open access at Springerlink.com

\begin{abstract}
Predicting the peak growth velocity in an individual patient with adolescent idiopathic scoliosis is essential or determining the prognosis of the disorder and timing of the (surgical) treatment. Until the present time, no accurate method has been found to predict the timing and magnitude of the pubertal growth spurt in the individual child. A mathematical model was developed in which the partial individual growth velocity curve was linked to the generic growth velocity curve. The generic curve was shifted and stretched or shrunk, both along the age axis and the height velocity axis. The individual age and magnitude of the PGV were obtained from the new
\end{abstract}

I. Busscher · F. H. Wapstra · A. G. Veldhuizen ( $\bowtie)$ Department of Orthopaedics, University Medical Center Groningen, University of Groningen, Hanzeplein 1, 9713 GZ Groningen, The Netherlands e-mail: a.g.veldhuizen@orth.umcg.nl

\section{Kingma}

Faculty of Human Movement Sciences, Research Institute MOVE, VU University Amsterdam, van der Boechorstraat 9, 1081 BT Amsterdam, The Netherlands

\section{R. de Bruin}

Department of High Performance Computing and Visualization, Donald Smits Center for Information Technology, University of Groningen, Nettelbosje 1, 9747 AJ Groningen,

The Netherlands

\section{G. J. Verkerke}

Department of Biomedical Engineering, University Medical Center Groningen, University of Groningen, Antonius Deusinglaan 1, 9713 AV Groningen, The Netherlands

\section{G. J. Verkerke}

Department of Biomechanical Engineering, University of Twente, Drienerlolaan 5, 7522 NB Enschede,

The Netherlands predicted complete growth velocity curve. Predictions were made using 2, 1.5, 1 and 0.5 years of the available longitudinal data of the individual child, starting at different ages. The predicted values of 210 boys and 162 girls were compared to the child's own original values of the PGV. The individual differences were compared to differences obtained when using the generic growth velocity curve as a standard. Using 2 years of data as input for the model, all predictions of the age of the PGV in boys and girls were significantly better in comparison to using the generic values. Using only 0.5 years of data as input, the predictions with a starting age from 13 to 15.5 years in boys and from 9.5 to 14.5 years in girls were significantly better. Similar results were found for the predictions of the magnitude of the PGV. This model showed highly accurate results in predicting the individual age and magnitude of the PGV, which can be used in the treatment of patients with adolescent idiopathic scoliosis.

Keywords Peak growth velocity · Prediction · Adolescent idiopathic scoliosis · Modeling

\section{Introduction}

The major difference between adult and pediatric medicine is that children still grow. Growth is a volumetric and longitudinal development and is essential to take into account when treating children with a wide variety of disorders. In particular, during the treatment of patients with adolescent idiopathic scoliosis (AIS), it is important to understand the growth and future growth of the patient. The prognosis of the curve progression and the timing of (surgical) treatment are mainly dependent on the timing of the pubertal growth spurt [1,2]. 
Several methods for prediction of the growth spurt or peak growth velocity (PGV) implicate the combination of different indicators of growth, such as the chronological age, skeletal age, Risser sign, age of menarche, etc. However, these methods are often only applicable to large groups of children and not accurate enough in predictions for the individual child [3-6]. Furthermore, many growth indicators, such as the skeletal age of the hand and wrist or the Tanner stage of sexual maturation need highly trained judgment to obtain an accurate assessment $[7,8]$.

A major difficulty in predicting growth of the individual child is the large variation in growth patterns between different children. The timing and magnitude of the pubertal peak growth spurt are highly variable. It is known, for example, that the PGV of total body height during puberty typically takes place between 10 and 14 years in $95 \%$ of the girls, and between 12 and 16 years in $95 \%$ of the boys. This range is too wide to allow for accurate predictions in the individual child. Furthermore, it is known that the magnitude of the peak growth is larger for individuals with early pubertal maturation as compared to those with late maturation [9-11]. So, phase and amplitude differences exist between individual children and therefore methods for prediction of the growth spurt in groups of children are not applicable to individuals.

While predictions of individual growth (velocity) curves are highly difficult to make, growth curves for the general population are available. Several models were used to produce representative generic growth and growth velocity curves taking the phase differences between individual children into account $[12,13]$.

In this study, it is proposed to apply a new mathematical model for prediction of the peak growth velocity in the individual child. The method fulfills the requirements of being easy to use, not expensive and generally applicable. It provides improved accuracy in predicting the timing and magnitude of the PGV of total body height; as such, it is useful for the treatment of patients with adolescent idiopathic scoliosis.

\section{Patients and methods}

It was assumed that a function $\mathrm{G}(t)$ for a generic growth curve and a function $\mathrm{V}(t)$ for a generic growth velocity curve are available. For the validation of the present growth model, the generic growth velocity curve for total body height of a Dutch population was chosen as described by Gerver and De Bruin [12], since the longitudinal growth data for validation of the model were obtained from Dutch children as well.

Using a custom-made program in Matlab ${ }^{\circledR}$ (Mathworks, Natick MA, USA), a smooth curve was fit through the available growth data of each individual child to obtain a smooth derivative, i.e., the partial growth velocity curve $v_{i}(t)$ of that particular child.

Next, a selected part of the original growth velocity curve of the child was linked to the known generic growth velocity curve by shifting and stretching or shrinking the generic growth velocity curve, both along the age axis ( $X$ axis) and along the height velocity axis ( $Y$-axis) by use of four parameters $\left(A_{i}, T_{i}, c_{i}\right.$, and $\left.k_{i}\right)$ :

$v_{i}(t)=A_{i} \mathrm{~V}\left(\left(t+c_{i}\right) / T_{i}\right)+k_{i}$

where $v_{i}(t)$ is the new predicted individual growth velocity curve, $c_{i}$ and $k_{\mathrm{i}}$ shift the generic growth curve $\mathrm{V}(t)$ along the $X$ and $Y$ axes, respectively, and $T_{i}$ and $A_{i}$ scale the generic growth curve along the $X$ and $Y$ axes, respectively (Fig. 1).

The parameters $A_{i}, T_{i}, c_{i}$ and $k_{i}$ were calculated for each individual child using a least squares optimization (Matlab function fmincon). Boundary conditions were applied to the four parameters to prevent outliers in the predictions (further considered in "Discussion").

By scaling the complete generic growth velocity curve, a new complete predicted individual growth velocity curve can be obtained (Fig. 1), even in cases where the individual child has not yet reached his or her PGV. From this new curve, the age of the PGV and the magnitude of the PGV can be abstracted by the program.

For validation of the model, the longitudinal growth data of total body height of 210 boys and 162 girls were used. The children were measured every 6 months in the last 4 years of primary school or the first 4 years of secondary school. In this way, two cohorts with longitudinal measurements of growth were obtained for children with ages ranging from 7.6 to 13.7 years; 11.0 to 17.7 years in boys

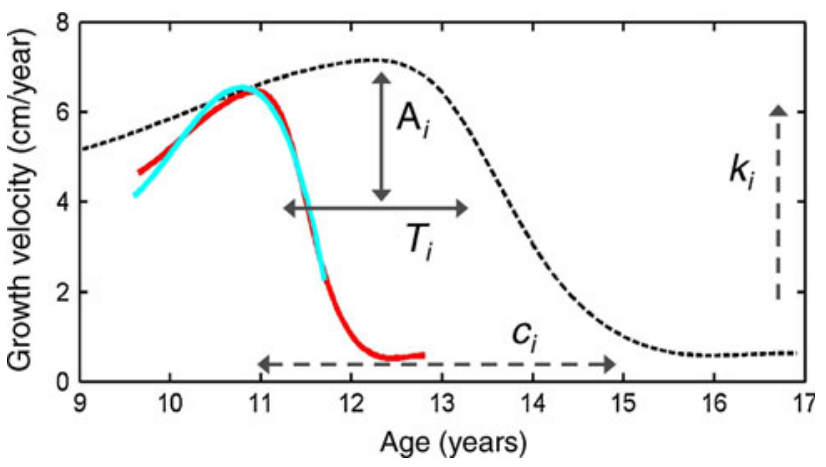

Fig. 1 Graphical representation of the predictive model. The original individual partial growth velocity curve (cyan line), the generic growth velocity curve (dashed black line), and the new individual predicted growth velocity curve (red line) are shown. $A_{i}$, scaling of the vertical amplitude of the growth velocity curve, $T_{i}$, scaling of the width of the velocity curve, $c_{i}$, shift of the growth velocity along the age axis, $k_{i}$, shift of the growth velocity curve along the growth velocity axis 
Table 1 Number of available children for each prediction of the age and magnitude of the PGV when using only $0.5,1,1.5$ and 2 years of the available data, respectively

\begin{tabular}{|c|c|c|c|c|c|c|c|c|c|c|c|c|c|c|c|c|c|c|}
\hline $\mathrm{N}$ & Starting age & 8 & 8.5 & 9 & 9.5 & 10 & 10.5 & 11 & 11.5 & 12 & 12.5 & 13 & 13.5 & 14 & 14.5 & 15 & 15.5 & 16 \\
\hline \multirow[t]{4}{*}{ Boys } & $\begin{array}{c}0.5 \text { year } \\
\text { data }\end{array}$ & & & 0 & 2 & 5 & 6 & 6 & 6 & 8 & 25 & 119 & 163 & 183 & 189 & 152 & 67 & 22 \\
\hline & 1 -year data & & & 0 & 2 & 5 & 6 & 6 & 7 & 4 & 26 & 118 & 161 & 176 & 149 & 67 & 22 & 6 \\
\hline & $\begin{array}{c}\text { 1.5-year } \\
\text { data }\end{array}$ & & & 0 & 2 & 5 & 6 & 5 & 2 & 3 & 25 & 116 & 154 & 136 & 64 & 22 & 6 & 2 \\
\hline & 2-year data & & & 0 & 2 & 5 & 5 & 1 & 2 & 2 & 24 & 111 & 114 & 51 & 19 & 6 & 2 & 0 \\
\hline \multirow[t]{4}{*}{ Girls } & $\begin{array}{c}0.5 \text {-year } \\
\text { data }\end{array}$ & 1 & 15 & 60 & 107 & 125 & 132 & 121 & 67 & 16 & 7 & 21 & 24 & 25 & 24 & 19 & & \\
\hline & 1-year data & 1 & 15 & 60 & 107 & 126 & 121 & 66 & 16 & 3 & 5 & 22 & 24 & 24 & 20 & 3 & & \\
\hline & $\begin{array}{c}\text { 1.5-year } \\
\text { data }\end{array}$ & 1 & 15 & 60 & 107 & 115 & 65 & 15 & 3 & 0 & 5 & 21 & 23 & 20 & 3 & 1 & & \\
\hline & 2-year data & 1 & 15 & 60 & 96 & 59 & 15 & 2 & 0 & 0 & 4 & 20 & 19 & 3 & 1 & 0 & & \\
\hline
\end{tabular}

Predictions in italics were significantly better for the age of the PGV and predictions in bold were significantly better for the magnitude of the $\mathrm{PGV}$ in comparison to the use of the generic values

and 7.7 to 13.4 years and 11.0 to 17.3 years in girls. Each child had measurements over a time span of at least 2 years (range 2.0-3.9 years, mean time span of boys 3.2 years, mean time span of girls 3.3 years).

Only growth curves of children were used in this validation study, which showed a clear pubertal growth spurt in that the highest peak was not the first or the last measurement. The original age and magnitude of the PGV were calculated beforehand by determining the highest peak in the derivative of the smoothed original individual growth curve.

The original individual age and magnitude of the PGV were compared to the values of the generic growth velocity curve, to determine the error that would have resulted when the generic growth velocity curve rather than the present model would have been used for predicting the age and magnitude of the PGV in each individual child.

Next, individual predictions were made with the model as described above using only $0.5,1.0,1.5$ and 2.0 years of the available data of the individual child as input, respectively. Furthermore, these predictions were made starting at different ages, with an interval of 0.5 years, from 9 to 16 years in boys and 8 to 15 years in girls.

The new predicted ages and magnitudes of the PGV of the individual child were compared to his or her own original age and magnitude of the PGV of total body height.

Subsequently, average absolute differences were calculated for the boys and girls to demonstrate the accuracy of the model.

For both the age and magnitude of the PGV, the absolute individual differences between the original and predicted values were compared to the individual differences between the predicted values and the values of the generic growth velocity curve, using paired-samples $T$ tests.

\section{Results}

The absolute difference between the individual ages of the PGV and the age of the PGV of the generic growth velocity curve was on average 0.66 years (SD 0.45) in boys and 1.08 years (SD 0.58) in girls. The magnitude of the PGV differed on average by $1.45 \mathrm{~cm} /$ year (SD 1.16) in boys and $1.59 \mathrm{~cm} /$ year (SD 1.13) in girls (Figs. 3, 4).

The number of available children for comparisons at each starting age and follow-up time are shown in Table 1. The results of the average absolute differences between the predicted and original age and magnitude of the PGV were shown when comparisons of more than 15 children were available. Therefore, only the results were shown from 12.5 to 16 years in boys and 8.5 to 15 years in girls, depending

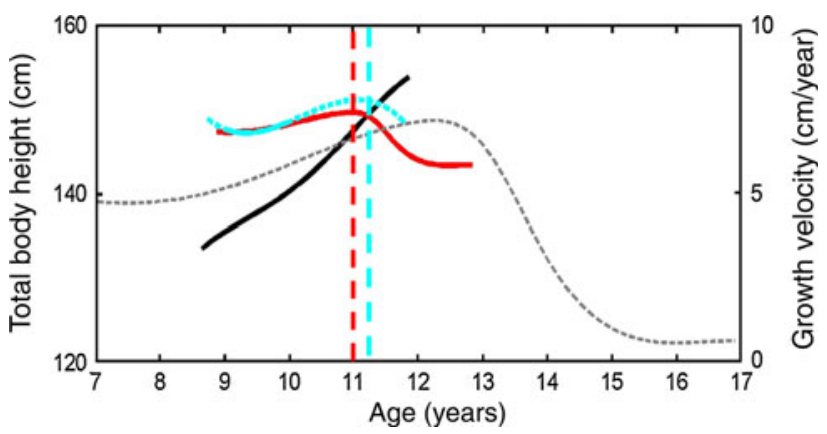

Fig. 2 Example of the prediction of a new individual growth velocity curve. The black curve represents the smooth fit of the original individual growth curve of total body height. The dashed cyan growth velocity curve of this individual contains a continuous part, which was used as input for the model. The gray dashed line represents the generic growth velocity curve from which the new, predicted individual growth velocity curve was constructed (red velocity curve). The vertical dashed lines represent the original (cyan) and new predicted (red) age of the PGV of this individual 

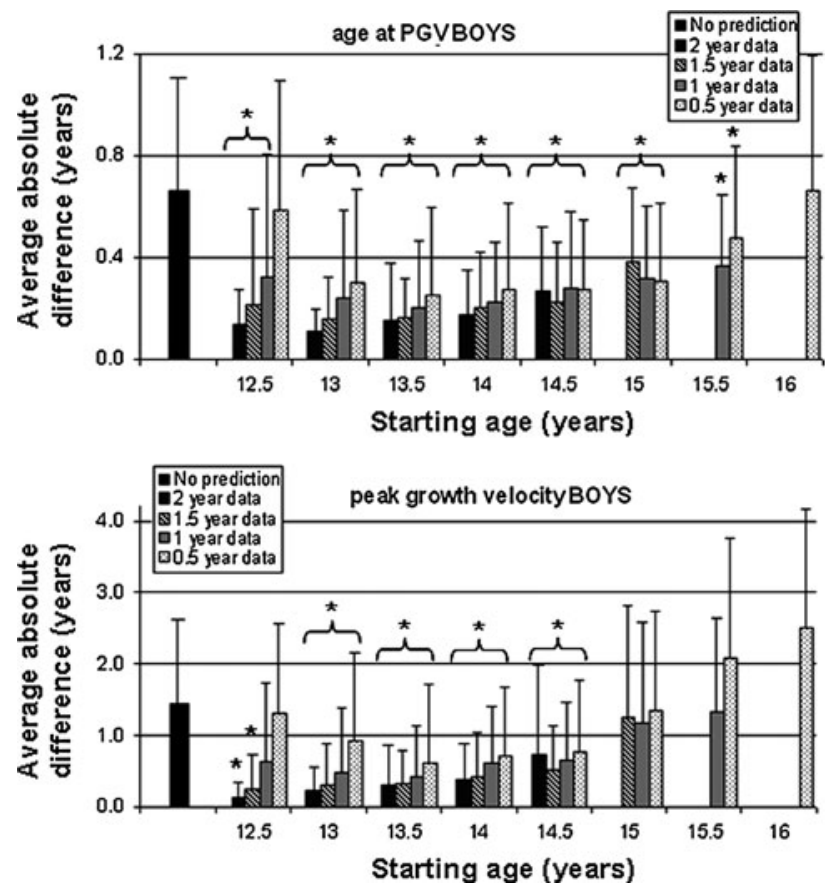

Fig. 3 Results of the average absolute differences between the original and predicted age of the PGV (upper graph) or magnitude of the PGV (lower graph) in boys. The thick black bar represents the average absolute difference between the values of the generic growth velocity curve and the original ages or magnitudes of the PGV of the individual children. The vertical bars represent the SD. Asterisks indicate significantly smaller difference between the prediction and the original age or magnitude of the PGV in comparison to the difference between the values of the generic growth velocity curve and the original age or magnitude of the PGV

on the amount of data used as input for the prediction (Figs. 3, 4).

An example of the prediction of the PGV of a girl is shown in Fig. 2. In this example, 1 year of available data points were used with a starting age of 9 years.

Results for all predictions of the age of the PGV are shown in Figs. 3 and 4.

Using 2 years of data as input for the model, the average absolute difference between the predicted age of the PGV and the original age of the PGV varied between 0.11 (SD 0.09 ) and 0.27 (SD 0.26) years in boys and between 0.15 (SD 0.20) and 0.70 (SD 0.54) years in girls, depending on the starting age. All these predictions were significantly better in comparison to using the generic values (Figs. 3, 4). When using only 0.5 years of the available data, the difference between the predicted and actual age of the PGV increased up to between 0.25 (SD 0.35) and 0.66 years (SD 0.53 ) in boys and between 0.27 (SD 0.34) and 1.17 (SD 0.50 ) years in girls (Figs. 3,4 ). Here, the predictions with a starting age ranging from 13 to 15.5 years in boys and from 9.5 to 14.5 years in girls were still significantly better in comparison to using the generic values of the growth velocity curve (Figs. 3, 4).
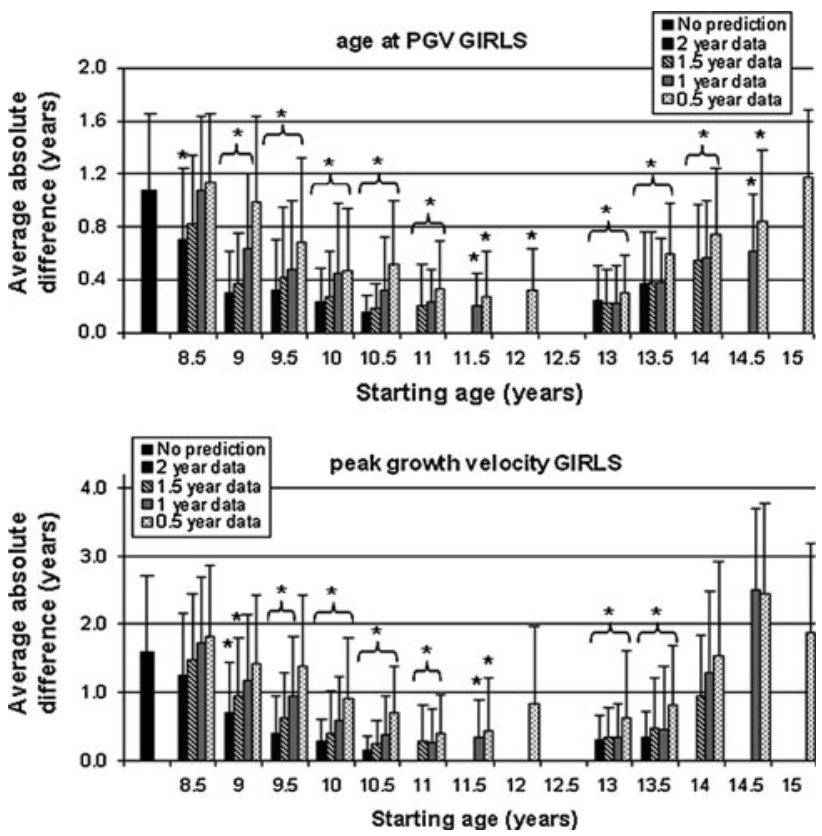

Fig. 4 Results of the average absolute differences between the original and predicted age of the PGV (upper graph) or magnitude of the PGV (lower graph) in girls. The thick black bar represents the average absolute difference between the values of the generic growth velocity curve and the original ages or magnitudes of the PGV of the individual children. The vertical bars represent the SD. Asterisks indicate significantly smaller difference between the prediction and the original age or magnitude of the PGV in comparison to the difference between the values of the generic growth velocity curve and the original age or magnitude of the PGV

The magnitude of the peak growth velocity differed on average between $0.14(\mathrm{SD} 0.22)$ and $0.73(\mathrm{SD} \mathrm{1.25)} \mathrm{cm} /$ year when using 2 years of data in boys, and between 0.15 (SD 0.20) and 1.24 (SD 0.93) cm/year in girls (Figs. 3, 4). In boys, all the predictions using 2 years of data were significantly better in comparison to using the values of the generic growth velocity curve. In girls, only the prediction starting from 8.5 years was not significantly better. When using only 0.5 years of data, the differences between the predicted and actual PGV increased up to between 0.60 (SD 1.12) and 2.51 (SD 1.65) $\mathrm{cm} /$ year in boys and between 0.40 (SD 0.56) and 2.46 (SD 1.31) $\mathrm{cm} /$ year in girls. The predictions starting from 13 to 14.5 years in boys and from 10 to 11.5 years and from 13 to 13.5 years in girls were still significantly better in comparison to using the generic growth velocity curve (Figs. 3, 4).

\section{Discussion}

This study demonstrated the utility of a new method for prediction of the peak growth velocity in the individual child. The analysis showed substantial improvement regarding the prediction of the age of the PGV, as well as 
the magnitude of the PGV of total body height, when compared with the use of a generic growth velocity curve. Previously, the orthopedic surgeon only knew that the pubertal growth spurt should appear around the age of 14 years in boys and 12 years in girls [7]. The present analysis showed that the predictions produced by this model were significantly better in most cases, even when using just a small amount of data points as input.

As mentioned in "Introduction", information regarding the peak growth velocity of the individual patient is highly important when discussing the prognosis and treatment of adolescent idiopathic scoliosis. Extensive research has been done regarding several maturity indicators and their relationship to curve progression in patients with idiopathic scoliosis. Many models need specialized training or are time consuming for the clinical practice. However, few methods have proven to be useful, like the simplified Tanner-Whitehouse-III system as described by Sanders et al. [14].

Although the theory behind this mathematical model can be somewhat difficult for clinicians, the program which is used is uncomplicated. The clinician should only fill in the available data points of length of the child, and the program calculates the predicted age and magnitude of the peak growth velocity. These values are the output of the program. Therefore, this program will be fast and easy to use for everyone. The person using the program will not have to calculate the actual mathematical formula.

A limitation of the present study was that the data were obtained from two cohorts of primary and secondary school children. Therefore, the growth curves with a clear pubertal growth spurt involving the age of around 12 years were less available. In particular in girls where the average growth spurt occurs at the age of 12.1 years; this resulted in small numbers of available children at starting ages ranging from 11.5 to 12.5 years (Fig. 4).

Other average results for different starting ages were not shown as well, since a cutoff point of at least 15 comparisons was chosen to be relevant for each prediction. However in boys, 12 out of 21 comparisons, with $N$ between 2 and 14, were still significantly better when using the model for predicting the age of the PGV in comparison to using the generic values. In girls, 7 out of 11 comparisons with $N$ between 2 and 14 were significantly better in predicting the age of the peak growth velocity of total body height (Table 1).

The validation of the model was performed with the data of healthy children without any signs of abnormal growth, since a reliable generic growth velocity curve was available for this population. The model should be further tested in children with adolescent idiopathic scoliosis. Although Veldhuizen et al. [15] and Duval-Beaupere [16, 17] failed to demonstrate any deviation of total body height or sitting height in scoliotic children in comparison to healthy children, the predictions of the model should be interpreted with care by the clinician treating these patients. The present study group is currently working on collecting extensive growth data of children with adolescent idiopathic scoliosis, but since collecting sufficient data takes several years, the model is first presented with already available growth data.

A limitation in the clinical use of the present model is that, when large errors are made in length measurements for the individual child, the results of the model prediction will not be accurate. Obviously, the influence of measurement errors will be greater when fewer measurements are performed in the individual child.

The accuracy of the PGV predictions was sensitive to the technique used to fit the original growth data of each child and to the selection of boundary conditions of the fitting parameters. While we presented the results using a fourth polynomial fit, we tested higher and lower order polynomials and a cubic spline fit as well. However, these results were always worse than for the fourth-order polynomial.

The second methodological factor that might influence the results was the selection of boundary conditions for $A_{i}$, $T_{i}, c_{i}$ and $k_{i}$. Pilot work showed that too wide boundaries resulted in a larger proportion of curves with obvious errors that could easily be identified through visual inspection. After removing these curves, the final results appeared to be insensitive to the selection of boundary conditions. In the final set of boundary conditions, $A_{i}$ was allowed to scale over a wide range, i.e., from 0.01 to 3 , but the model did not reach these values. $T_{i}$ was restricted in its scaling from 0.4 to 1.2 . $c_{i}$ was restricted mainly in the positive direction from -4 to +1.25 , and $k_{i}$ was restricted from 0 to +4 to avoid negative growth velocities.

An advantage of the present prediction model is that it can theoretically be applied to different body length measures. Besides the growth spurt of total body length, the sitting height is also important for the treatment of AIS. Furthermore, knowing the timing and magnitude of the PGV of subischial leg length, for example, is essential when treating patients with leg length differences. Therefore, the model should be further validated for prediction of the age and magnitude of the PGV of other body length dimensions.

This study showed the results of the validation of a new mathematical model for prediction of the peak growth velocity in the individual child. The model showed accurate results in predicting the individual age of the $P G V$ as well as the magnitude of the PGV of total body height, which can be used in the treatment of patients with adolescent idiopathic scoliosis, as well as other orthopedic pediatric disorders. 
Acknowledgments This study was supported by the Dutch Technology Foundation, STW, applied science division of NWO and the Technology Program of the Ministry of Economic Affairs.

Open Access This article is distributed under the terms of the Creative Commons Attribution Noncommercial License which permits any noncommercial use, distribution, and reproduction in any medium, provided the original author(s) and source are credited.

\section{References}

1. Sanders JO, Little DG, Richards BS (1997) Prediction of the crankshaft phenomenon by peak height velocity. Spine 22:1352-1356

2. Sanders JO, Browne RH, McConnell SJ et al (2007) Maturity assessment and curve progression in girls with idiopathic scoliosis. J Bone Joint Surg Am 89:64-73

3. Little DG, Song KM, Katz D et al (2000) Relationship of peak height velocity to other maturity indicators in idiopathic scoliosis in girls. J Bone Joint Surg Am 82:685-693

4. Sanders JO, Browne RH, Cooney TE et al (2006) Correlates of the peak height velocity in girls with idiopathic scoliosis. Spine 31:2289-2295

5. Sanders JO (2007) Maturity indicators in spinal deformity. J Bone Joint Surg Am 89(1):14-20

6. Wang S, Qiu Y, Ma Z et al (2007) Histologic, risser sign, and digital skeletal age evaluation for residual spine growth potential in Chinese female idiopathic scoliosis. Spine 32:1648-1654
7. Coleman L, Coleman J (2002) The measurement of puberty: a review. J Adolesc 25:535-550

8. Dimeglio A (2001) Growth in pediatric orthopaedics. J Pediatr Orthop 21:549-555

9. Gerver WJ, de Bruin R (2003) Growth velocity: a presentation of reference values in Dutch children. Horm Res 60:181-184

10. Tanner JM, Whitehouse RH, Marubini E et al (1976) The adolescent growth spurt of boys and girls of the Harpenden growth study. Ann Hum Biol 3:109-126

11. Tanner JM, Davies PS (1985) Clinical longitudinal standards for height and height velocity for North American children. J Pediatr 107:317-329

12. Gerver WJ, de Bruin R (2001) Paediatric morphometrics: a reference manual. 2nd edn. UPM Maastricht

13. Tanner JM (1978) Foetus into man. Physical growth from conception to maturity. OBP London

14. Sanders JO, Khoury JG, Kishan S et al (2008) Predicting scoliosis progression from skeletal maturity: a simplified classification during adolescence. J Bone Joint Surg 90:540-553

15. Veldhuizen AG, Baas PC, Webb PJ (1986) Observations on the growth of the adolescent spine. J Bone Joint Surg Br 68:724-728

16. Duval-Beaupere G (1970) Pathogenic relationship between scoliosis and growth. In: Zorab PA (ed) Scoliosis and growth, vol 1. Churchill Livingstone, Edinburg, pp 58-64

17. Duval-Beaupere G, Combes J (1971) Upper and lower segments in the physiological growth of girls. Long-term study of the growth of 54 girls. Arch Fr Pediatr 28:1057-1071 